rapport à l'académie nationale de chirurgie dentaire complications infectieuses
des prothèses articulaires
et infection bucco-dentaire synthèse des données bibliographiques actuelles

RÉSUMÉ La pose de prothèses articulaires est un acte chirurgical de plus en plus courant. Les chirurgiens orthopédistes redoutent une infection péri-articulaire d'origine hématogène. Les examens pré-opératoires demandés comportent toujours actuellement la recherche d'éventuels foyers dentaires. L'odontologiste peut avoir des difficultés à rédiger le certificat exigé par le chirurgien.

C'est pour cette raison que l'Académie Nationale de

Michel GUILLAIN

Ancien Président

de l'Académie Nationale

de Chirurgie Dentaire.

Bernard TOMENO

Jean-Pierre COURPIED

Chirurgiens orthopédistes.

Yves COMMISSIONAT

Membre de l'Académie Nationale

de Chirurgie Dentaire.

Florine BOUKHOBZA

Membre de l'Académie Nationale de Chirurgie Dentaire.

\section{N. AL-ZRIQAT}

Docteur en chirurgie dentaire. Chirurgie Dentaire a pensé qu'une mise au point sur ce sujet était nécessaire. Un rapport lui a été remis.

Le lecteur trouvera ci-joint un condensé de ce rapport qui pourra être consulté in extenso dans le $n^{\circ} 2006.49 \mathrm{du}$ Bulletin de l'Académie Nationale de Chirurgie Dentaire. 


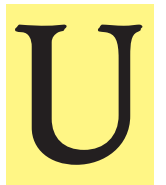

$\mathrm{n}$ des développements majeurs de la chirurgie de l'après-guerre fut le remplacement des articulations gravement endommagées par des prothèses articulaires (fig. 1a, 1 b, fig. 2).

À l'heure actuelle, la pratique de la pose de prothèses articulaires est courante.

Néanmoins, les complications infectieuses au niveau des prothèses articulaires restent un problème.

En effet, les conséquences peuvent être

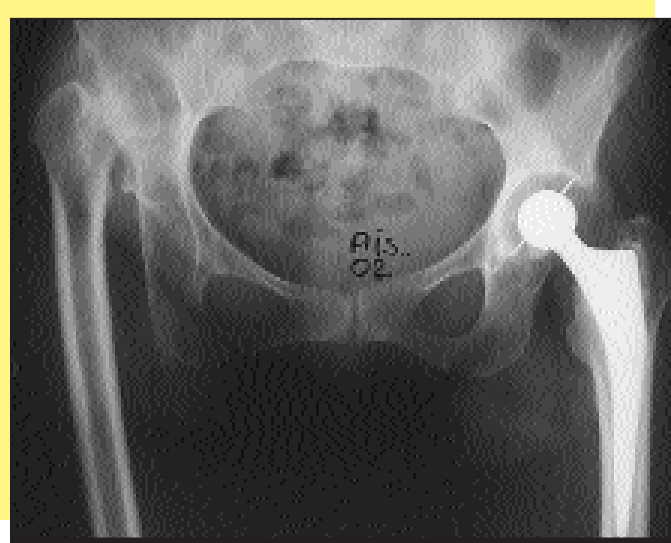

fig. 1a Luxation congénitale de la hanche droite. Prothèse de la hanche gauche, cimentée sans anomalie. désastreuses. On peut être amené à remplacer la prothèse si cela est possible. Dans le cas contraire, après le retrait de la prothèse sans remplacement, une pseudarthrose survient et provoque une déficience squelettique, un racourcissement des membres et une détérioration physique importante (Salvati, 1984).

Dans le pire des cas, l'infection d'une prothèse articulaire peut aboutir à une ostéomyélite chronique voire à une amputation ou à la mort.

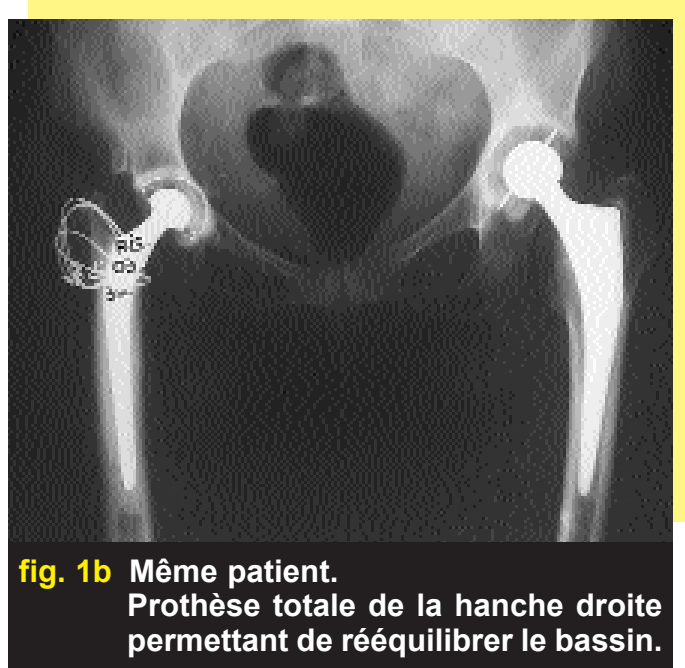

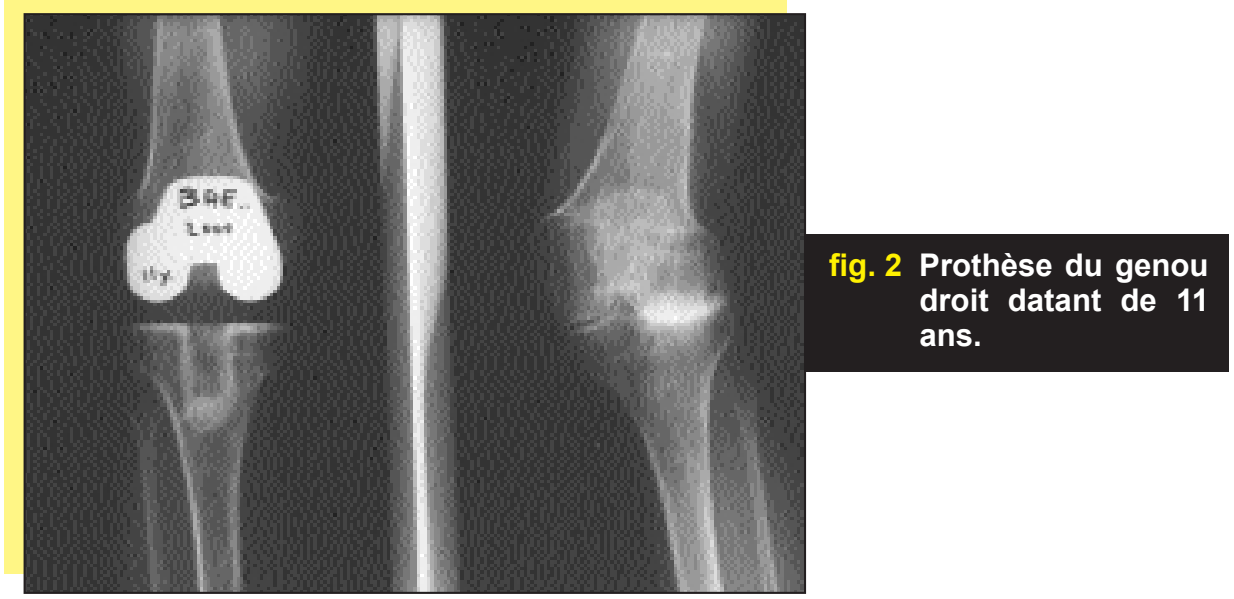


Nous pouvons distinguer les infections opératoires et les infections hématogènes.

- Les infections opératoires sont, soit d'expression précoce dans les deux mois suivant l'intervention, soit d'expression plus chronique dans l'année voire les deux années qui suivent l'intervention.

Elles sont dues à la contamination per-opératoire qui, elle-même, peut être liée à des conditions d'intervention ou à des foyers infectieux latents chez le patient.

Grâce à l'utilisation de l'antibioprophylaxie pré-opératoire et à l'évolution des techniques, leur fréquence a baissé depuis ces vingt dernières années.

- Les infections hématogènes sont des infections produites à partir d'un foyer à distance de l'articulation opérée.

Ces infections hématogènes peuvent survenir sans aucun délai de temps après la réalisation de l'arthroplastie.

Elles proviennent de sites à distance : infections bucco-dentaires, urinaires, de la sphère ORL, respiratoires, cutanées ou autres.

Chirurgiens orthopédistes, chirurgiens dentistes et stomatologistes sont parfaitement conscients de ce risque, mais il est difficile à évaluer, beaucoup plus que pour l'endocardite infectieuse.

Cette différence d'évaluation provient de plusieurs facteurs.
L'endocardite survient chez des patients présentant une cardiopathie préalable, la pose de prothèses valvulaires ne fait qu'accroître le risque. Par contre, la pose de prothèses articulaires s'effectue souvent sur des sujets ne présentant aucune pathologie générale.

En outre, si la preuve bactériologique est souvent difficile à évaluer en présence d'une endocardite, elle l'est infiniment plus en présence d'une infection autour d'une prothèse articulaire.

Ces faits rejaillissent sur les rapports entre chirurgiens orthopédistes et chirurgiens dentistes et stomatologistes.

Les premiers demandent aux seconds de rechercher et d'éliminer tout foyer dentaire infectieux patent ou latent. Les seconds sont pris entre deux feux : élargir les indications et supprimer, outre les foyers évidents, tout foyer qui n'est que suspect et ainsi édenter peutêtre trop largement et inutilement un patient, ou bien être plus économes et voir en cas d'infection articulaire, leur responsabilité mise en cause.

Trois catégories de patients doivent être distinguées :

- recherche de foyers infectieux d'origine dentaire avant pose d'une prothèse articulaire ;

- traitements dentaires ou survenue d'une infection bucco-dentaire chez un sujet porteur d'une prothèse articulaire ;

- infection autour d'une prothèse articulaire. Recherche d'une éventuelle étiologie bucco-dentaire. 


\section{recherche de foyers infectieux d'origine dentaire avant pose d'une prothèse articulaire}

La conférence de consensus qui eut lieu en 1997 entre l'American Dental Association et l'American Academy of Orthopaedic Surgeons conseille simplement :

«Les patients qui doivent subir la pose d'une prothèse articulaire devraient avoir une bonne santé dentaire avant l'intervention et devraient être encouragés à faire effectuer des soins dentaires si nécessaires».

Ces directives sont peu précises.

Il nous faut distinguer deux catégories de patients : les patients sans risque, les patients à risque.

\section{patients sans risque}

Ce sont des patients qui, en dehors de leur pathologie articulaire, ne présentent aucune pathologie reconnue.

Il n'y a aucune contre-indication aux actes dentaires non sanglants :

\section{Bilan pré-opératoire}

Un principe essentiel doit être retenu. Les interventions articulaires sont bien souvent des interventions non urgentes. Ce fait donne toute latitude au praticien pour effectuer les traitements conservateurs, après, bien évidemment, une concertation avec le chirurgien orthopédiste.

Il faut assurer et maintenir une hygiène bucco-dentaire en donnant les conseils nécessaires.

Le traitement des lésions constatées est décrit plus loin.
Seul figure dans ce chapitre, l'attitude vis-à-vis des dents dépulpées :

- dent dépulpée à canaux parfaitement traités depuis plus d'un an et ne présentant aucune lésion périapicale. Descrozailles ne considère pas comme un foyer infectieux une telle dent ;

- dent dépulpée à canaux non totalement traités depuis plus d'un an sans lésion péri-radiculaire (cf. plus loin reprise des traitements radiculaires).

\section{Directives opératoires}

Il existe deux catégories d'actes : non sanglants, sanglants.

\section{- Actes non sanglants :}

Ils ne sont pas contre-indiqués.

Les traitements endodontiques posent un problème particulier : il faut les réaliser, si possible, au moins trois mois avant l'intervention articulaire. Extraire la dent si des complications infectieuses aiguës surviennent; si un début de lésion apicale apparaît : résection apicale pour les mono-radiculées, extraction pour les pluri-radiculées.

Reprises de traitements radiculaires : directives équivalentes.

\section{- Actes sanglants :}

Étant donné que le sujet est indemne de tare, les indications des traitements antibiotiques n'ont aucune particularité.

Un bain de bouche à base de chlorexidine est recommandable avant l'acte opératoire. 
Les principales interventions sont les suivantes :

- extraction des dents irrécupérables, des dents incluses à sac péricoronaire ouvert ;

- la chirurgie péri-apicale doit être réalisée au moins 3 mois avant la pose de la prothèse articulaire. $\mathrm{La}$ dent sera conservée s'il existe après ce délai une ébauche de calcification, sinon elle doit être extraite ;

- les amputations radiculaires, les transplantations, la réimplantation, la chirurgie parodontale sont contre-indiquées en raison des incertitudes pronostiques ;

- la pose d'implants est également contre-indiquée, car l'apparition d'une péri-implantite est imprévisible. Par contre, un implant posé depuis plus d'un an, parfaitement intégré, avec une sertissure gingivale normale peut être conservé.

Le bilan pré-opératoire sera réévalué en fonction du tableau clinique et du suivi bucco-dentaire.

\section{patients à risque}

Figurent dans cette catégorie les affections suivantes : diabète de type 1 et 2 , hémophilie, dénutrition, cancer en évolution, insuffisance rénale, hépatique en fonction $\mathrm{du}$ bilan biologique, immuno-dépression constitutionnelle acquise, ou d'origine thérapeutique, SIDA en trithérapie, prise de médicaments au longs cours (corticothérapie, AINS, chimiothérapie...), polyarthrite rhumatoïde, lupus érythémateux, valvulopathies (ces dernières ont leurs propres indications thérapeutiques).

\section{Bilan et directive opératoires}

Les directives sont évidemment beaucoup plus strictes.

Les règles suivantes peuvent s'appliquer aux dents dépulpées :

- dents dépulpées à canaux parfaitement traités : à conserver selon les normes de Descrozailles ;

- dents dépulpées à canaux non totalement traités : extraction même en l'absence de lésions péri-apicales ;

- reprise des traitements radiculaires : contre-indiquée.

\section{Actes non sanglants}

Les traitements endodontiques seront également réalisés, si possible, trois mois avant l'intervention articulaire. Ils se dérouleront sous champ opératoire étanche (digue), en une séance, sur des canaux parfaitement accessibles (principalement les monoradiculées), sous couverture antibiotique.

\section{Actes sanglants}

Ces actes nécessiteront une antibioprophylaxie.

La chirurgie péri-apicale est contreindiquée. Les indications des extractions sont très larges. 


\section{traitements dentaires ou survenue d'une infection bucco-dentaire chez un sujet porteur d'une prothèse articulaire}

Deux conférences de consensus entre l'American Dental Association et l'American Academy of Orthopaedic Surgeons ont été consacrées à ce sujet en 1997 et en 2003.

La deuxième conférence approfondit les problèmes d'hygiène dentaire: brossage rigoureux des dents, hygiène des espaces interdentaires, hydropulseurs.

Il semble que la période critique pour l'ensemencement d'une articulation soit celle des deux années qui suivent l'intervention.

Il faut donc ajouter à la liste des patients à risque :

- les patients opérés depuis moins de deux ans ;

- les patiens ayant des antécédents d'infection articulaire sur prothèse.

De nombreuses études statistiques ont été effectuées.

Pour Seymour, sur 100000 patients porteurs d'une prothèse articulaire, seulement 30 développent une infection nécessitant son remplacement. Jacobson et coll. confirment cette notion. Le risque infectieux est minime : 29,3 cas pour 1000000 d'actes dentaires. Thyne et Ferguson ont relevé dans la littérature 21 cas d'infection articulaire éventuellement d'origine dentaire. Dans 3 cas seulement un streptocoque viridens a été décelé dans l'articulation. Wahl est encore plus incisif dans un article intitulé «Les mythes de l'infec- tion des prothèses articulaires d'origine dentaire». Il ne retient que moins de 25 cas documentés prouvant ces relations.

La rareté de ces cas provoque, surtout chez les auteurs américains, un rejet de la prophylaxie antibiotique. Les risques de l'antibiothérapie sont bien connus : troubles digestifs, toxicité, allergie, développement, sélection et transmission d'une résistance microbienne. Ils insistent non seulement sur le rapport risque/bénéfice mais aussi sur le rapport coût/efficacité.

Skyest et Coykendall citent le Concil of Dental Therapeutics : «Les faits sont actuellement insuffisants pour soutenir la nécessité ou l'efficacité d'une prophylaxie antibiotique chez les patients porteurs d'une prothèse articulaire qui doivent subir des soins dentaires». Pour certains, le risque de la prescription (y compris le risque de décès) est plus grand que le risque d'une infection articulaire.

D'autres auteurs paraissent moins hostiles envers l'antibioprophylaxie. Leur opinion se fonde sur le résultat d'enquêtes menées auprès des divers praticiens. Odontologistes et chirurgiens orthopédistes semblent d'accord sur la nécessité d'une antibioprophylaxie lors d'actes dentaires invasifs. Ce rapport soutient ce deuxième point de vue. 


\section{CONSÉQUENCES CLINIQUES}

Quelle doit donc être l'attitude de l'odontologiste ou du stomatologiste lorsqu'il doit pratiquer un acte ou traiter un accident infectieux chez un sujet porteur d'une prothèse articulaire ?

\section{ANTIBIOPROPHYLAXIE}

Elle semble rejetée par de nombreux auteurs en raison de son éventuelle inefficacité et des effets secondaires parfois graves. Il faut noter que les auteurs anglo-saxons paraissent beaucoup plus inquiets de la gravité de ses effets secondaires que les praticiens européens.

Il semble en réalité qu'il soit préférable de la prescrire devant tout phénomène infectieux d'origine bucco-dentaire.

En ce qui concerne les interventions, elle serait inutile, sauf pour les interventions complexes ou de longue durée (au-delà de 45 minutes).

Cette attitude est valable pour les patients sans risque. Les indications des antibiotiques pour les patients à risque sont beaucoup plus larges.

\section{NATURE DE L'INTERVENTION}

Il faut là encore distinguer les patients à risque des patients sans risque.

\section{Pour les patients sans risque}

- Les traitements endodontiques pourront être réalisés sous couverture antibiotique sur des dents à canaux parfaitement accessibles. Extraction au moindre incident. La reprise de traitements radiculaires n'est pas souhaitable.

- Anesthésie locale, détartrages, sondages peuvent être réalisés à l'exclusion de la chirurgie parodontale. La chirurgie péri-apicale sera également exclue.

\section{Pour les patients à risque}

- Les dents dépulpées à canaux parfaitement traités pourront être conservées selon les normes de Descrozailles. Les traitements endo dontiques sont risqués, les extractions ont des indications très larges.

\section{problèmes infectieux}

\section{autour d'une prothèse articulaire recherche d'une éventuelle étiologie bucco-dentaire}

Un certain nombre d'études bactériologiques ont été effectuées au niveau du site articulaire (fig. 3a, 3b, 3c). Pour Skiest, la plupart de ces infections sont dues aux staphylocoques, suivies par le corynebacterium, les streptocoques $\beta$ hémolytiques et les anaérobies. Les streptocoques viridens ne sont retenus que dans $2 \%$ des cas et seulement $0,4 \%$ de ces infections reconnaissent une étiologie dentaire.

Pour Seymour et Whitworth, plus de $66 \%$ des infections articulaires sont provoquées par le staphylocoque (essentiellement d'origine cutanée) et seulement $4,9 \%$ par le streptocoque 


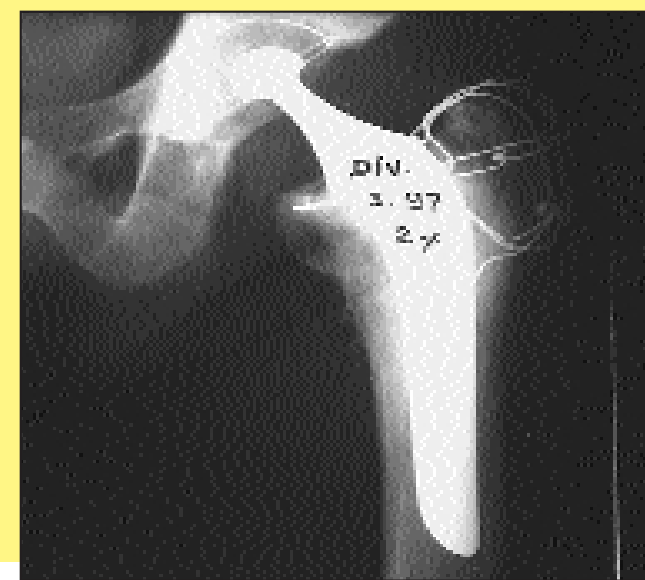

fig. 3a Prothèse de la hanche datant de 2 ans. Situation en 1997. Aucune anomalie.

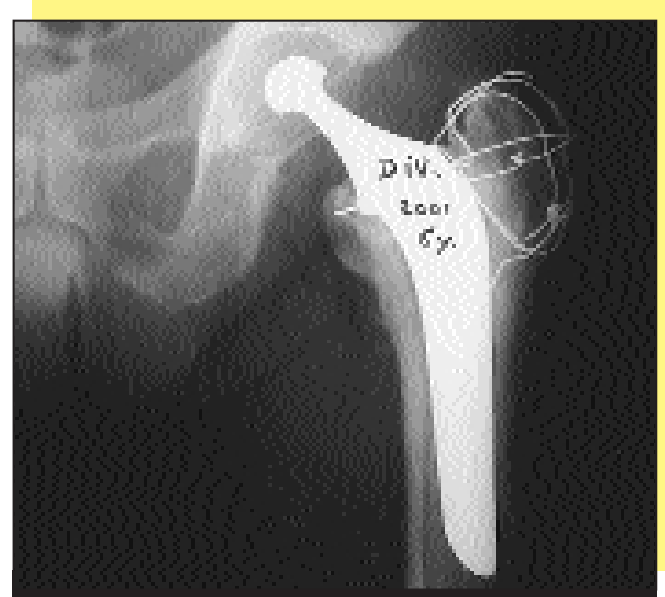

fig. 3b Même observation. Situation en 2001. Descellement septique par infection à distance. Existence d'un liseré ciment/os.

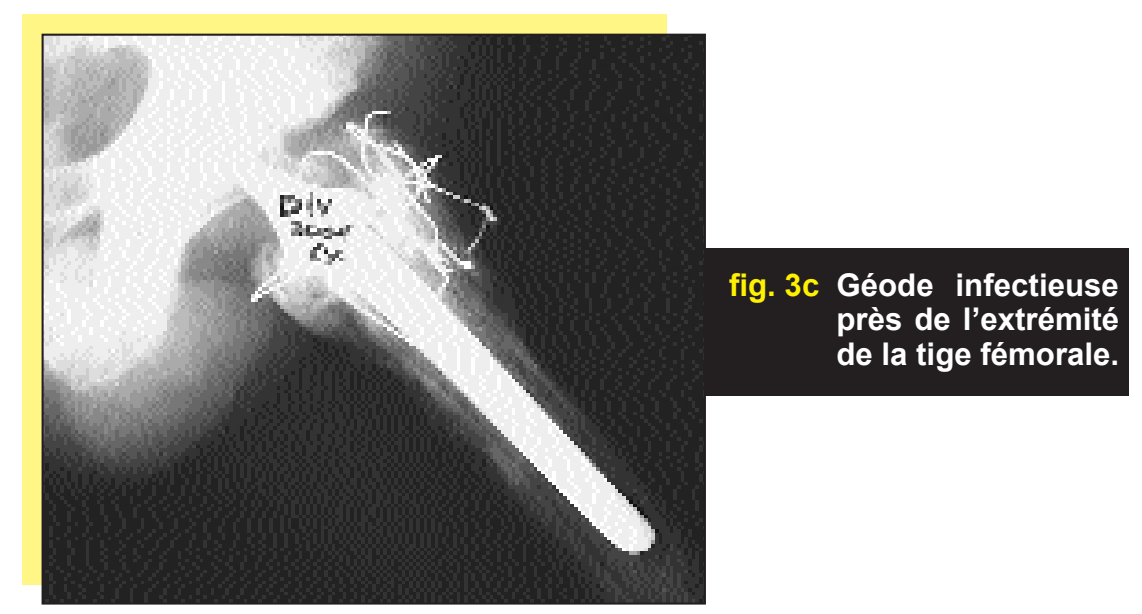

viridens d'éventuelle origine buccale. Ces auteurs citent une revue de 21 cas d'infections articulaires attribuables à un acte dentaire. Chez un de ces patients, le même micro-organisme fut retrouvé dans les cultures provenant de la salive, du sang et de la prothèse articulaire. Il s'agissait d'un streptocoque $\beta$ hémolytique. Sur une étude de 1000 patients, seulement 3 présentèrent une infection articulaire.
Or, 224 d'entre eux avaient subi des actes dentaires invasifs sans antibioprophylaxie.

Une autre étude incrimine principalement une infection de la peau et des tissus mous.

Sur 110 cas, quatre purent être attribués au streptocoque viridens. Ces quatre patients avaient été victimes d'une infection dentaire aiguë récente. 
La revue de la littérature semble ne permettre de relever qu'une seule étude française, celle de Rougerie. Ses conclusions sont les suivantes: «Si certains auteurs ont décrit des cas cliniques d'infections tardives de prothèse totale de hanche, avec de fortes présomptions d'origine bucco-dentaire, aucun n'en a réellement rapporté la preuve absolue».

La couverture antibiotique sera prescrite en accord avec le chirurgien orthopédiste.

\section{CONSÉQUENCES ODONTO-STOMATOLOGIQUES}

L'infection autour d'une protèse articulaire exige un examen bucco-dentaire minutieux.

Faut-il extraire les dents dépulpées ?

Étant donné le peu de fréquence de l'étiologie dentaire prouvée, les dents à canaux parfaitement traités, sans lésion apicale pourront être conservées. Dans les autres cas, l'extraction s'impose.

Il faudra surtout profiter de ces extractions pour effectuer une étude bactériologique des dents extraites. La technique du prélèvement par immersion de l'apex devrait être appliquée (Lepoivre, Commissionat, Chikhani et coll.). Nous rappelons brièvement ici le principe de cette technique. Le site de prélèvement choisi est le delta apical, particulièrement riche en germes (fig. 4). Après désinfection de la dent extraite par immersion dans de l'alcool iodé, l'apex est sectionné à l'aide d'une forte pince coupante (fig. 5 et 6 ) et mis en culture dans un tube (fig. 7).

Cette technique permet de comparer les germes recueillis au niveau de la prothèse articulaire avec ceux recueillis au niveau de l'apex et de confirmer une éventuelle identité.
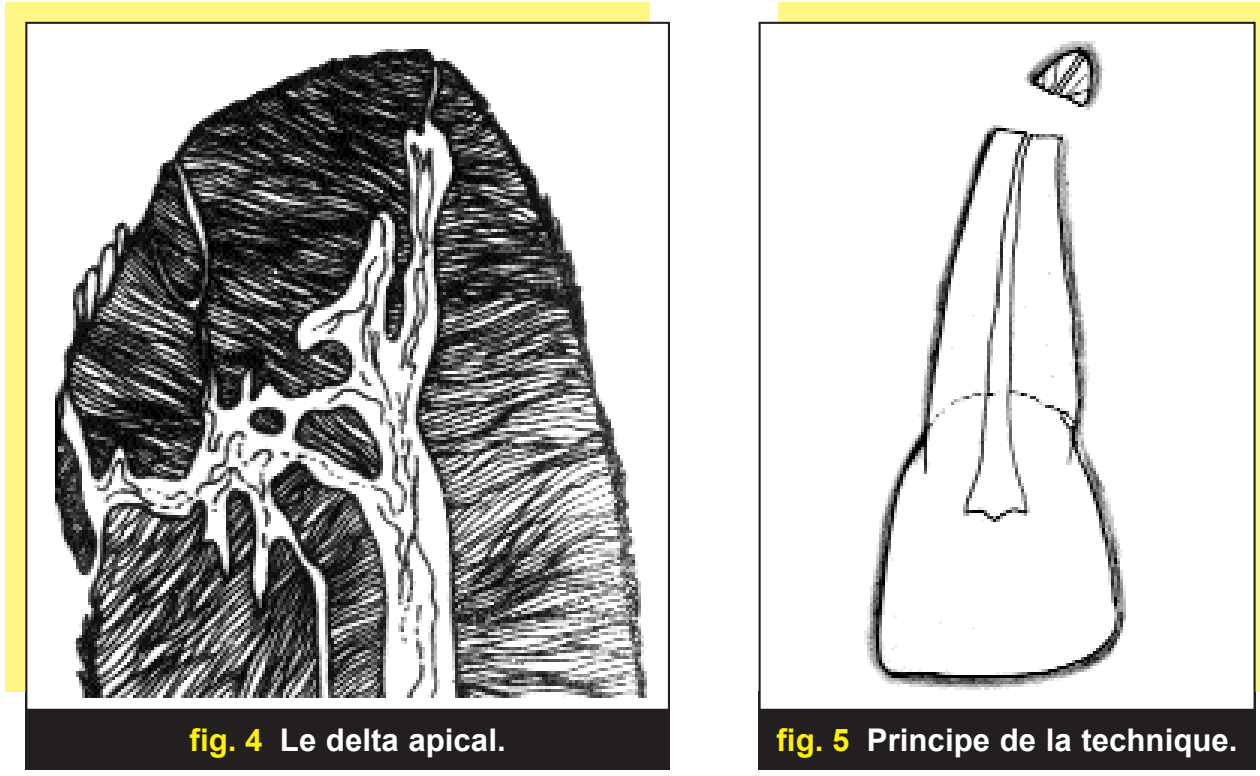

fig. 5 Principe de la technique. 

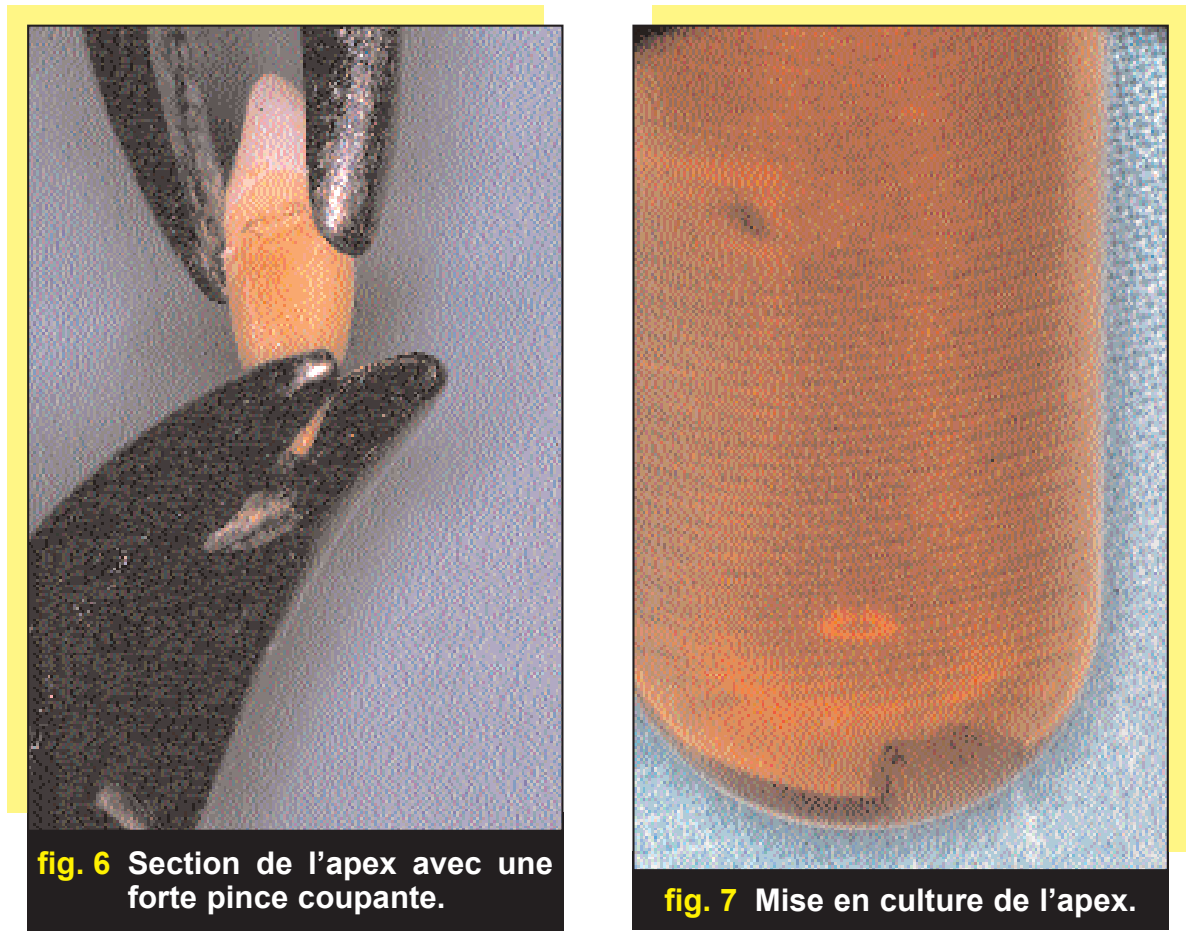

\section{considérations thérapeutiques générales}

Nous avons pu constater les réserves émises par beaucoup d'auteurs américains concernant l'antibioprophylaxie. Néanmoins, quand les antibiotiques sont prescrits, principalement chez les sujets à risque, la conférence de consensus américaine propose le schéma suivant pour encadrer des interventions dentaires.

\begin{tabular}{|l|l|l|}
\hline \multicolumn{1}{|c|}{ Situation } & \multicolumn{1}{|c|}{ Antibiotique } & \multicolumn{1}{c|}{ et voie d'administration } \\
\hline Prophylaxie standard & $\begin{array}{l}\text { Cephalexine, cephradine } \\
\text { ou amoxicilline }\end{array}$ & $\begin{array}{l}\text { 2 g per os une heure avant } \\
\text { le geste opératoire }\end{array}$ \\
\hline Voie orale inutilisable & $\begin{array}{l}\text { Cefalozine } \\
\text { ou amoxicilline }\end{array}$ & $\begin{array}{l}\text { Cefalozine } 1 \mathrm{~g} \text { ou amoxicilline } 2 \mathrm{~g} \\
\text { IM ou IV une heure avant le geste } \\
\text { opératoire }\end{array}$ \\
\hline $\begin{array}{l}\text { Allergie } \\
\text { aux } \beta \text { lactamines }\end{array}$ & Clindamycine & $\begin{array}{l}600 \mathrm{mg} \text { per os une heure avant } \\
\text { le geste opératoire }\end{array}$ \\
\hline $\begin{array}{l}\text { Allergie } \\
\text { aux } \beta \text { lactamines } \\
\text { et voie orale inutilisable }\end{array}$ & Clindamycine & $\begin{array}{l}600 \mathrm{mg} \mathrm{IV} \mathrm{une} \mathrm{heure} \mathrm{avant} \\
\text { le geste opératoire }\end{array}$ \\
\hline
\end{tabular}


Une seconde dose n'est pas recommandée, sauf en cas d'infection.

Il nous semble en France plus sage de s'inspirer des travaux émis par les conférences de consensus en 1992 et 2002 sur la prophylaxie de l'endocardite infectieuse :

\begin{tabular}{|l|l|l|}
\hline \multicolumn{1}{|c|}{ Situation } & \multicolumn{1}{c|}{ Antibiotique } & \multicolumn{1}{c|}{$\begin{array}{c}\text { Posologie } \\
\text { et voie d'administration }\end{array}$} \\
\hline $\begin{array}{l}\text { Pas d'allergie } \\
\text { aux } \beta \text { lactamines }\end{array}$ & Amoxicilline & $\begin{array}{l}3 \mathrm{~g} \text { per os, prise unique } \\
\text { dans l'heure précédent le geste }\end{array}$ \\
\hline $\begin{array}{l}\text { Allergie } \\
\text { aux } \beta \text { lactamines }\end{array}$ & $\begin{array}{l}\text { Pristinamycine ou } \\
\text { Clindamycine }\end{array}$ & $\begin{array}{l}1 \mathrm{~g} \text { per os, } \\
600 \mathrm{mg} \text { per os, } \\
\text { prise unique dans l'heure } \\
\text { précédant le geste }\end{array}$ \\
\hline
\end{tabular}

Il faut adjoindre à l'antibioprophylaxie une antisepsie locale préopératoire sous la forme de bain de bouche à la chlorexidine.

En postopératoire, un suivi de la température sera demandé à l'opéré dès le lendemain matin et les jours suivants. En cas de température anormalement élevée, le patient devra revoir l'orthopédiste en consultation. De toute façon, l'opéré sera revu le lendemain de l'intervention. En cas de cicatrisation normale, aucun traitement n'est à envisager. En cas de cicatrisation retardée, déficiente, avec caillot en voie de nécrose associée à : fétidité, douleurs vives, œdème périmaxillaire, une antibiothérapie complémentaire est mise en place selon la conférence de consensus.
«Lors de certains gestes invasifs en un lieu infecté, il sera nécessaire de prolonger l'antibiothérapie».

En plus de $3 \mathrm{~g}$ d'amoxicilline une heure avant le geste opératoire, on complètera par $1 \mathrm{~g}$ toutes les 8 heures soit $3 \mathrm{~g}$ sur plusieurs jours jusqu'à la guérison de la plaie opératoire :

- élimination de toute zone nécrotique suspecte ;

- disparition de toute fétidité ;

- disparition d'un œdème périmaxillaire.

Le contrôle de la température devra indiquer un retour à la normale sinon l'orthopédiste sera consulté.

En cas d'allergie aux bêtalactamines, clindamycine ou pristinamycine seront utilisées selon les mêmes principes.

\section{conclusions}

Pour les auteurs américains les infections sur prothèses articulaires sont rares. L'étiologie dentaire est encore plus rare. Lorsque l'infection sur prothèse articulaire survient, il est difficile de pouvoir prouver la filiation entre cette infection et une étiologie dentaire. Les germes retrouvés au niveau de la prothèse articulaire sont rarement des germes considérés comme facteurs d'infection dentaire.

C'est pourquoi, les règles strictes édictées pour l'endocardite infectieuse doi- 
vent être conservées en matière de pathologie des prothèses articulaires.

Il semble que pour les patients ne présentant aucune autre pathologie que leur pathologie articulaire, ces règles peuvent être adoucies selon les schémas énoncés plus haut.

Par contre, chez les sujets à risque elles doivent conserver toute leur vigueur.
Lorsque l'infection articulaire est déclarée, il est bien évident que les règles sévères seront appliquées. Des extractions sont en général nécessaires. Il serait bon alors d'utiliser la technique du prélèvement par immersion de l'apex pour mettre en évidence les germes retrouvés et les comparer avec ceux décelés au niveau de la prothèse articulaire. 